\title{
Horocycles in hyperbolic 3-manifolds
}

\section{Citation}

McMullen, Curtis T, Amir Mohammadi, and Hee Oh. 2015. Horocycles in hyperbolic 3-manifolds. Working paper, Harvard University.

\section{Published Version}

10.1007/s00039-016-0373-8

\section{Permanent link}

http://nrs.harvard.edu/urn-3:HUL.InstRepos:24981204

\section{Terms of Use}

This article was downloaded from Harvard University's DASH repository, and is made available under the terms and conditions applicable to Other Posted Material, as set forth at http:// nrs.harvard.edu/urn-3:HUL.InstRepos:dash.current.terms-of-use\#LAA

\section{Share Your Story}

The Harvard community has made this article openly available.

Please share how this access benefits you. Submit a story.

\section{Accessibility}




\title{
Horocycles in hyperbolic 3-manifolds
}

\author{
Curtis T. McMullen, Amir Mohammadi and Hee Oh
}

22 November 2015

\section{Contents}

1 Introduction . . . . . . . . . . . . . . . . . . 1

2 Background ....................... 4

3 Configuration spaces and double cosets . . . . . . . . . 6

4 Moving to the renormalized frame bundle . . . . . . . 8

5 Exceptional frames . . . . . . . . . . . . . . . 10

6 Classification of $U$-orbit closures . . . . . . . . . . . . . . 11

$7 \quad$ Classification of $A U$-orbit closures . . . . . . . . . . . 13

Research supported in part by the Alfred P. Sloan Foundation (A.M.) and the NSF. 


\section{Introduction}

Let $M=\Gamma \backslash \mathbb{H}^{3}$ be a complete hyperbolic 3-manifold. A horocycle $\chi \subset M$ is an isometrically immersed copy of $\mathbb{R}$ with zero torsion and geodesic curvature 1. The torsion condition means the $\chi$ lies in an immersed totally geodesic plane.

One can regard $\chi$ as a limit of planar circles whose centers have moved off to infinity. It is natural to ask what the possibilities are for its closure,

$$
\bar{\chi} \subset M \text {. }
$$

When $M$ has finite volume, it is well-known that strong rigidity properties hold; e.g. $\bar{\chi}$ is always an immersed homogeneous submanifold of $M$ [Sh], $[\mathrm{Rn}]$. Continuing the investigation begun in [MMO], this paper shows that rigidity persists for horocycles in certain infinite volume 3-manifolds. These are the first examples of Zariski dense groups $\Gamma \subset \operatorname{Isom}\left(\mathbb{H}^{3}\right)$, other than lattices, where the topological behavior of horocycles in $\Gamma \backslash \mathbb{H}^{3}$ has been fully described.

Horocycles in acylindrical manifolds. To state the main results, recall that the convex core of $M$ is given by:

$$
\operatorname{core}(M)=\Gamma \backslash \operatorname{hull}(\Lambda) \subset M,
$$

where $\Lambda \subset \widehat{\mathbb{C}}$ is the limit set of $\Gamma$, and $\operatorname{hull}(\Lambda) \subset \mathbb{H}^{3}$ is its convex hull. We say $M$ is a rigid acylindrical manifold if its convex core is a compact, proper submanifold of $M$ with totally geodesic boundary. Our first result describes the behavior of horocycles in $M$.

Theorem 1.1 Let $\chi \subset M=\Gamma \backslash \mathbb{H}^{3}$ be a horocycle in a rigid acylindrical 3-manifold. Then either:

1. $\chi \subset M$ is a properly immersed 1-manifold; or

2. $\bar{\chi} \subset M$ is a properly immersed 2-manifold, equidistant from a totally geodesic surface $S \subset M$; or

3. $\bar{\chi}$ is the entire 3-manifold $M$.

Corollary 1.2 The closure of any horocycle is a properly immersed submanifold of $M$. 
Similar results for geodesic planes in $M$ are obtained in [MMO].

Homogeneous dynamics. To make Theorem 1.1 more precise, we reformulate it in terms of the frame bundle $\mathrm{F} M \rightarrow M$.

Let $G$ denote the simple, connected Lie group $\mathrm{PGL}_{2}(\mathbb{C})$. Within $G$, we have the following subgroups:

$$
\begin{aligned}
& H=\mathrm{PSL}_{2}(\mathbb{R}), \\
& A=\left\{\left(\begin{array}{cc}
a & 0 \\
0 & a^{-1}
\end{array}\right): a \in \mathbb{R}_{+}\right\} \text {, } \\
& K=\mathrm{SU}(2) /( \pm I) \text {, } \\
& N=\left\{n_{s}=\left(\begin{array}{ll}
1 & s \\
0 & 1
\end{array}\right): s \in \mathbb{C}\right\} \text {, } \\
& U=\left\{n_{s}: s \in \mathbb{R}\right\} \text {, and } \\
& V=\left\{n_{s}: s \in i \mathbb{R}\right\} \text {. }
\end{aligned}
$$

Upon identifying $\mathbb{H}^{3}$ with $G / K$, we obtain the natural identifications

$$
\mathrm{F} M \cong \Gamma \backslash G \text { and } \quad M \cong \Gamma \backslash G / K .
$$

Every (oriented) horocycle $\chi$ in $M$ lifts to a unique unipotent orbit $x U$ in the frame bundle F $M$. Let $A_{+}$be the positive semigroup in $A$, defined by $a \geq 1$, and let

$$
\mathrm{RF}_{+} M=\left\{x \in \mathrm{F} M: \overline{x A_{+}} \subset \mathrm{F} M \text { is compact }\right\} .
$$

This locus is closed and invariant under $A N$.

Our main result may now be stated as follows (see $\S 6$ ).

Theorem 1.3 Let $M=\Gamma \backslash \mathbb{H}^{3}$ be a rigid acylindrical 3-manifold. Then for any $x \in \mathrm{F} M$, either

1. $x U$ is closed;

2. $\overline{x U}=x v H v^{-1} \cap \mathrm{RF}_{+} M$ for some $v \in V$; or

3. $\overline{x U}=\mathrm{RF}_{+} M$.

It is readily verified that these three alternatives give the three cases in Theorem 1.1, using the fact that the map FM $\rightarrow M$ is proper and its restriction to $\mathrm{RF}_{+} M$ is surjective. 
Corollary 1.4 The closure of any $U$-orbit in $\mathrm{RF}_{+} M$ is homogeneous, in the sense that

$$
\overline{x U}=x S \cap \mathrm{RF}_{+} M
$$

for some closed subgroup $S \subset G$ with $U \subset S$.

Indeed, we can take $S=U, v H v^{-1}$ or $G$. As we will see in $\S 7$, the classification of $A U$-orbits follows from Theorem 1.3 as well:

Corollary 1.5 For any $x \in \mathrm{RF}_{+} M$, we have $\overline{x A U}=\overline{x H} \cap \mathrm{RF}_{+} M$.

The possibilities for $\overline{x H}$ are recalled in Theorem 2.3 below. (For $x \notin \mathrm{RF}_{+} M$, it is easy to see that the orbit $x A U$ is closed.)

Strategy. The mechanism behind the proof of Theorems 1.3 is the following dichotomy. Suppose a horocycle $\chi \subset M$ limits on a properly embedded, totally geodesic surface $S$ (such as one of the boundary components of the convex core of $M$ ). If $\chi$ is contained in $S$ then $\chi$ is trapped and $\bar{\chi}=S$; otherwise, $\chi$ is scattered by $S$, and $\bar{\chi}=M$. In both cases the behavior of $\chi$ is strongly influenced by the behavior of the horocycle flow on $S$. To complete the proof we show that, up to the action of $V$, every recurrent horocycle accumulates on such a surface $S$. This step uses the classification of $H$-orbits from [MMO].

We remark that any connected subgroup of $G$ generated by unipotent elements is conjugate to $N, H$ or $U$. Theorem 1.3 completes the description of the topological dynamics of these groups acting on $\mathrm{F} M$, since the behavior of $H$ and $N$ was previously known (see $\S 2$ ).

Outline of the paper. The remainder of the paper is devoted to the proof of Theorem 1.3. In $\S 2$ we review existing results about dynamics on FM. In $\S 3$ we establish a general lemma about the double coset space $U \backslash G / H$, and in $\S 4$ we prove an approximation theorem for $U$-orbits. The space of exceptional frames is introduced in $\S 5$, and the proof of Theorem 1.3 is completed in $\S 6$. Corollary 1.5 is deduced in $\S 7$.

Remark: General acylindrical manifolds. When $M$ is a convex cocompact, acylindrical manifold that is not rigid, the behavior of horocycles can be radically different from the rigid case. For example, a horocycle orthogonal to a closed leaf of the bending lamination of $\partial \operatorname{core}(M)$ can be properly embedded, giving rise to a frame $x \in \mathrm{F} M$ with a compact $A$-orbit and a nonrecurrent $U$-orbit. The scattering argument also breaks down, due to the lack of totally geodesic surfaces in $M$. It is an open problem to develop a rigidity theory for these and other infinite-volume hyperbolic 3-manifolds. 


\section{Background}

In this section we introduce notation and recall known results regarding topological dynamics on FM.

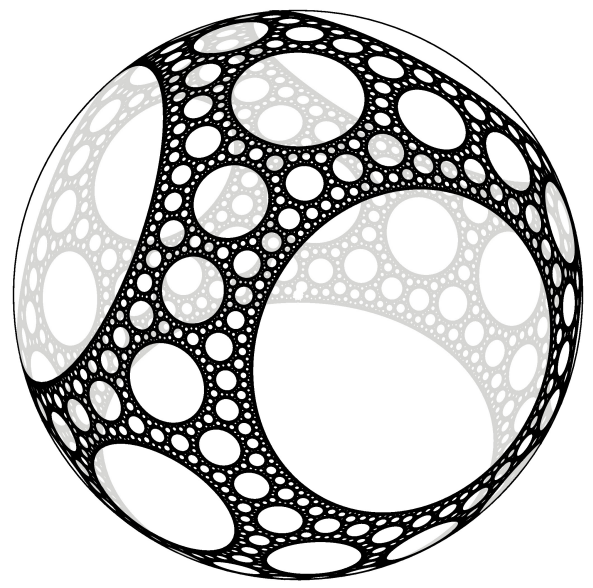

Figure 1. Limit set of a rigid acylindrical manifold.

Geometry on $\mathbb{H}^{\mathbf{3}}$. Notation for $G$ and its subgroups was introduced in $\S 1$. We also let $A_{\mathbb{C}}=\left\{\left(\begin{array}{cc}z & 0 \\ 0 & z^{-1}\end{array}\right): z \in \mathbb{C}^{*}\right\}$. The action of $G$ on $\mathbb{H}^{3}=G / K$ extends continuously to a conformal action of $G$ by Möbius transformations on the Riemann sphere,

$$
\widehat{\mathbb{C}}=\mathbb{C} \cup\{\infty\} \cong G / A_{\mathbb{C}} N,
$$

and the union $\mathbb{H}^{3} \cup \widehat{\mathbb{C}} \cong B^{3}$ is compact. We let $\widehat{\mathbb{R}}=\mathbb{R} \cup\{\infty\}$ denote the standard circle on $\widehat{\mathbb{C}}$. Its orientation-preserving stabilizer in $G$ is $H$.

Let $M=\Gamma \backslash \mathbb{H}^{3}$ be a hyperbolic 3-manifold. The natural covering map

$$
\mathrm{FH} H^{3} \cong G \rightarrow \mathrm{F} M \cong \Gamma \backslash G
$$

will be denoted by $g \mapsto[g]$. The limit set of $\Gamma$ is characterized by $\Lambda=\widehat{\mathbb{C}} \cap \overline{\Gamma p}$, for any $p \in \mathbb{H}^{3}$; the domain of discontinuity is its complement, $\Omega=\widehat{\mathbb{C}}-\Lambda$. The convex hull of $\Lambda$ is the smallest convex subset of $\mathbb{H}^{3}$ containing all geodesics with both endpoints in the limit set; and its quotient gives the convex core of $M$ :

$$
\operatorname{core}(M)=\Gamma \backslash \operatorname{hull}(\Lambda) \subset M \text {. }
$$


A group is elementary if it contains an abelian subgroup with finite index. We will always assume that $\Gamma \cong \pi_{1}(M)$ is a nonelementary group.

Surfaces in $\boldsymbol{M}$. There is a natural correspondence between

(i) Closed $H$-orbits in F $M$,

(ii) Properly immersed, totally geodesic surfaces $S \subset M$, and

(iii) Circles $C \subset \widehat{\mathbb{C}}$ such that $\Gamma C$ is discrete in the space of all circles, $\mathcal{C} \cong G / H$.

This correspondence is given, with suitable orientation conventions, by $C=$ $[x H], S=$ the projection of hull $(C) \subset \mathbb{H}^{3}$ to $M$, and $x H=\mathrm{T} S$, the bundle of frames tangent to $S$.

Convex cocompact manifolds. Now assume that the convex core of $M$ is compact. The renormalized frame bundle of $M$ is defined by

$$
\mathrm{RF} M=\{x \in \mathrm{F} M: \overline{x A} \subset \mathrm{F} M \text { is compact }\} .
$$

Replacing $A$ with $A_{+}$in the definition above, we obtain the locus $\mathrm{RF}_{+} M$. Note that $\mathrm{RF} M$ is invariant under $A$ and $\mathrm{RF}_{+} M$ is invariant under $A N$.

In terms of the universal cover, we have $[g] \in \mathrm{RF}_{+} M$ if and only if $g(\infty) \in \Lambda$, while $[g] \in \operatorname{RF} M$ if and only if $\{g(0), g(\infty)\} \subset \Lambda$.

Minimality. We now turn to some dynamical results. Let $L$ be a closed subgroup of $G$. We say $X \subset \mathrm{F} M$ is an $L$-minimal set if $\overline{x L}=X$ for all $x \in X$.

Theorem 2.1 (Ferte) If $M$ is convex cocompact, then the locus $\mathrm{RF}_{+} M$ is an $N$-minimal set.

See [Fer, Cor. C(iii)]; a generalization appears in [Win]. We also record the following result from [Da]:

Theorem 2.2 (Dal'bo) If $\Gamma \subset H$ is a nonelementary convex cocompact Fuchsian group, then $(\Gamma \backslash H) \cap \mathrm{RF}_{+} M$ is a $U$-minimal set.

Rigid acylindrical manifolds. Recall that $M$ is a rigid acylindrical manifold if $M$ is convex cocompact, of infinite volume, and $\partial$ core $(M)$ is totally geodesic. In this case $\Omega \subset \widehat{\mathbb{C}}$ is the union of a dense set of round disks with disjoint closures, and $\Lambda$ is a Sierpiǹski curve; see Figure 1.

Theorem 2.3 Let $M$ be a rigid acylindrical manifold. Then for any $x \in$ $\mathrm{RF} M$, either $x H$ is closed or $\overline{x H}=\left(\mathrm{RF}_{+} M\right) H$. 
Proof. Since $\Omega$ is a union of round disks, any circle that meets $\Lambda$ in just one point can be approximated by a circle meeting $\Lambda$ in two or more points; thus

$$
\overline{(\mathrm{RF} M) H}=\left(\mathrm{RF}_{+} M\right) H .
$$

Let $H^{\prime}=\mathrm{PGL}_{2}(\mathbb{R})=H \cup j H$, where $j=\left(\begin{array}{cc}1 & 0 \\ 0 & -1\end{array}\right)$. Note that $A j=j A$ and hence $(\operatorname{RF} M) j=\operatorname{RF} M$.

With $H^{\prime}$ in place of $H$, Theorem 2.3 is proved in [MMO, Cor 1.7]. Using the $H^{\prime}$ version, we can conclude that either $x H$ is closed or $\overline{x H^{\prime}}=$ $\left(\mathrm{RF}_{+} M\right) H^{\prime}$. In the latter case, $\mathrm{RF} M$ is contained in $\overline{x H} \cup \overline{x H} j$. But $\mathrm{RF} M$ has a dense $A$-orbit [MMO, Thm 4.3], so RFM is contained in $\overline{x H}$ or $\overline{x H} j$. In either case, we have

$$
\mathrm{RF} M=(\mathrm{RF} M) j \subset \overline{x H} j^{2}=\overline{x H} .
$$

Hence $\overline{x H}=\left(\mathrm{RF}_{+} M\right) H$ by equation (2.1) above.

\section{Configuration spaces and double cosets}

This section and the next present two self-contained results that will be used in $\S 6$ below. In this section we will prove:

Theorem 3.1 Suppose $g_{n} \rightarrow$ id in $G-V H$, and $T_{n} \subset U$ is a sequence of $K$-thick sets. Then there is a $K^{\prime}$-thick set $V_{0} \subset V$ such that

$$
\lim \sup T_{n} g_{n} H \supset V_{0} .
$$

Double cosets. As motivation for the Theorem, we remark that the double coset space $U \backslash G / H$ is the moduli space of pairs $(\chi, P) \subset \mathbb{H}^{3}$, where $\chi$ is a horocycle and $P \cong \mathbb{H}^{2}$ is a hyperplane. This moduli space is highly nonseparated near the identity coset, where $\chi \subset P$. This means that as $\chi$ approaches $P$, the pair $(\chi, P)$ can have many different limiting configurations, depending on how we choose coordinates. The Theorem above describes, more precisely, the different limiting configurations that arise. The appearance of multiple configurations is a basic mechanism at work in homogeneous dynamics.

Limits of sets. We recall that the limsup of a sequence of sets $X_{n} \subset G$ consists of all limits of the form $g=\lim x_{n_{k}}$, where $n_{k} \rightarrow \infty$ and $x_{n_{k}} \in X_{n_{k}}$.

Thick sets and polynomials. We say $T \subset \mathbb{R}$ is $K$-thick if

$$
[1, K] \cdot|T|=[0, \infty)
$$


This notion also makes sense for $T$ inside any Lie group isomorphic to $\mathbb{R}$, such as $U$ or $V$. A basic fact about thick sets, which will be used below, is the following. Let $p \in \mathbb{R}[x]$ be a polynomial of degree $d$, and let $T \subset \mathbb{R}$ be $K$-thick. Then for any symmetric interval $I=[-a, a] \subset \mathbb{R}$, we have

$$
\max _{x \in T \cap I}|p(x)| \geq k \max _{x \in I}|p(x)|,
$$

where $k>0$ depends only on $K$ and $d$. For more details, see [MMO, $\S 8$ ].

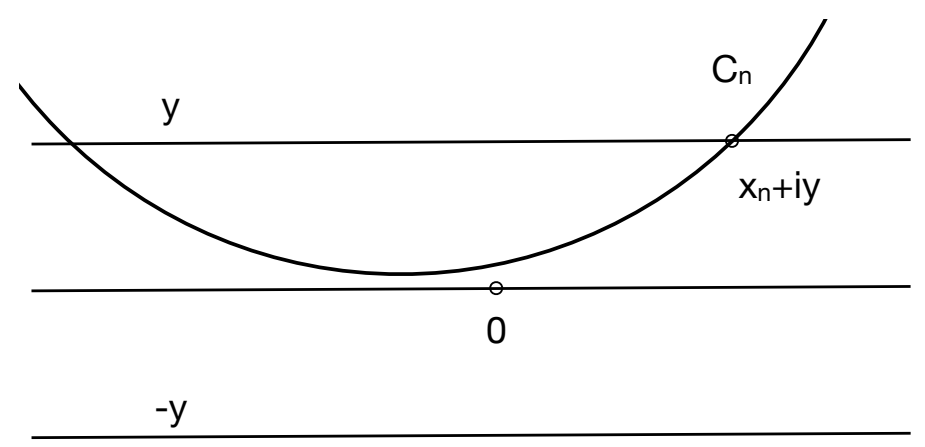

Figure 2. The circles $C_{n} \rightarrow \widehat{\mathbb{R}}$ eventually meet the locus $|\operatorname{Im}(z)=y|$.

Proof of Theorem 3.1. Fix $y>0$. We will first show that $\lim \sup U g_{n} H$ contains $v$ or $v^{-1}$, where $v(z)=z+i y$.

Let $C_{n}=g_{n}(\widehat{\mathbb{R}})$. Since $g_{n} \rightarrow \mathrm{id}$, we have $C_{n} \rightarrow \widehat{\mathbb{R}}$ in the Hausdorff topology on closed subsets of $\widehat{\mathbb{C}}$. Note that for $n \gg 0, C_{n} \cap \mathbb{C}$ is either a circle of large radius or a straight line of nonzero slope (since $g_{n} \notin V H$ ), Thus $C_{n}$ meets the locus $L=|\operatorname{Im}(z)|=y$ for all $n \gg 0$. Passing to a subsequence, we can assume that $C_{n} \cap L \neq \emptyset$ for all $n$, and that the point of $C_{n} \cap L$ closest to the origin has the form $x_{n}+\epsilon y$ for a fixed $\epsilon= \pm 1$ (see Figure 2). Let $u_{n}(z)=z-x_{n}$; then

$$
u_{n} g_{n}(\widehat{\mathbb{R}}) \rightarrow \widehat{\mathbb{R}}+i \epsilon y
$$

as $n \rightarrow \infty$. It follows that $u_{n} g_{n} h_{n}(z) \rightarrow z+i \epsilon y$ for suitable $h_{n} \in H$, since the latter group can be used to reparameterize $\widehat{\mathbb{R}}$. Equivalently, $v$ or $v^{-1}$ belongs to $\lim \sup U g_{n} H$.

We now take into account the thick sets $T_{n}$. Note that at the scale $\left|x_{n}\right|$, the arc of $g_{n}(\widehat{\mathbb{R}})$ close to $\mathbb{R}$ is well-modeled by a parabola, i.e. the graph of a quadratic polynomial. Applying equation (3.1) to this polynomial, we find there is a $K^{\prime}$ depending only on $K$, and a sequence $x_{n}^{\prime}+i y_{n}^{\prime} \in C_{n}$, such that 
$u_{n}^{\prime}(z)=z-x_{n}^{\prime} \in T_{n}$, and $1 \leq\left|y / y_{n}^{\prime}\right| \leq K^{\prime}$. Passing to a subsequence and arguing as above, we conclude that $v(z)=z+i y^{\prime}$ belongs to $\lim \sup T_{n} g_{n} H$ for some $y^{\prime}$ with $1 \leq\left|y / y^{\prime}\right| \leq K^{\prime}$. Since $y>0$ was arbitrary, this shows that $V \cap\left(\lim \sup T_{n} g_{n} H\right)$ is a $K^{\prime}$-thick subset of $V$.

Remark. Theorem 3.1 is a strengthening of [MMO, Lemma 8.2]; the proof here is more geometric.

\section{Moving to the renormalized frame bundle}

In this section we describe how to use $U$ to move points close to RF $M$ into RFM. The boundary of the convex core of $M$ gives rise to an exceptional case.

Theorem 4.1 Suppose $x_{n} \in(\operatorname{RF} M) U$ and $x_{n} \rightarrow y \in \operatorname{RF} M$. There there exists a sequence $u_{n} \in U$ such that $x_{n} u_{n} \in \operatorname{RF} M$ and

1. We have $u_{n} \rightarrow \mathrm{id}$, and hence $x_{n} u_{n} \rightarrow y$; or

2. There is a component $S$ of $\partial \operatorname{core}(M)$ such that $y H=\mathrm{T} S$, and $x_{n} u_{n}$ accumulates on $\mathrm{T} S$ as $n \rightarrow \infty$.

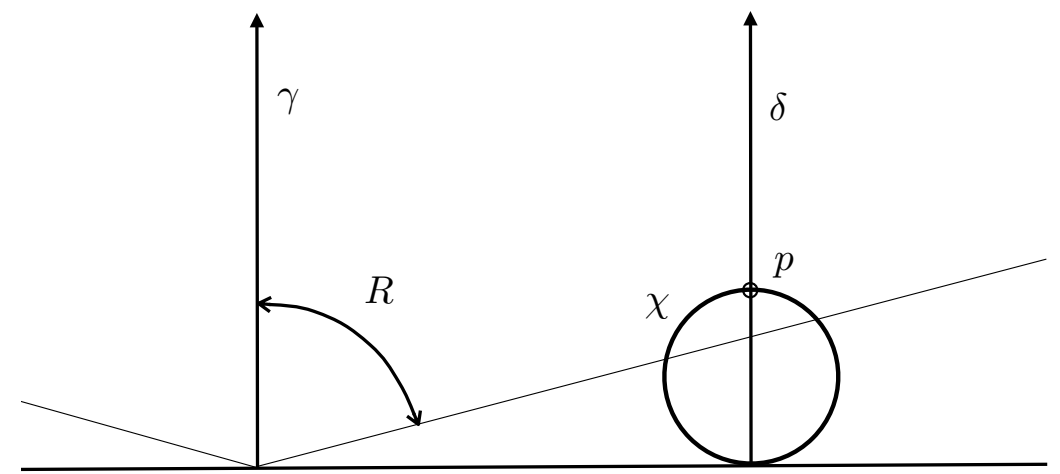

Figure 3. If $d(\gamma, \chi) \leq R-1$, with $R \gg 0$, then $d(p, \gamma)<R$.

The proof relies on the following fact from planar hyperbolic geometry.

Lemma 4.2 Let $\gamma, \chi \subset \mathbb{H}$ be a geodesic and a horocycle respectively, let $\delta$ be a geodesic joining the base of $\chi$ to one of the endpoints of $\gamma$, and let $\{p\}=\delta \cap \chi$. Then for all $R \gg 0$, if $d(\chi, \gamma)<R-1$, then $d(p, \gamma)<R$. 
The proof is indicated in Figure 3, where the endpoint in common to $\gamma$ and $\delta$ is at infinity. Note that an $R$-neighborhood of $\gamma \subset \mathbb{H}$, for $R \gg 0$, is bounded by a pair of rays meeting at an angle of nearly $180^{\circ}$.

Proof of Theorem 4.1. Choose $g_{n} \rightarrow g_{0}$ in $G$ such that $\left[g_{n}\right]=x_{n}$ and $\left[g_{0}\right]=y$, and let $C_{n}=g_{n}(\widehat{\mathbb{R}})$.

Recall that $[g] \in \mathrm{RF} M$ if and only if $\{g(0), g(\infty)\} \subset \Lambda$. By assumption, $g_{n}(\infty) \in \Lambda$ for all $n$, and $g_{0}(0) \in \Lambda$. Moreover, since $x_{n} \in(\operatorname{RF} M) U$, there exist $s_{n} \in \mathbb{R}$ such that $g_{n}\left(s_{n}\right) \in \Lambda$. Let us arrange that $\left|s_{n}\right|$ is as small as possible; then $g_{n}\left(I_{n}\right) \subset \Omega$, where $I_{n}=\left(-s_{n}, s_{n}\right)$. Setting $u_{n}(z)=z-s_{n}$, we then have $\left[g_{n} u_{n}\right]=x u_{n} \in \operatorname{RF} M$.

It remains to verify that (1) or (2) is true. If $\left|s_{n}\right| \rightarrow 0$, then clearly we are in case (1), so let us assume that $s=\lim \sup \left|s_{n}\right|>0$. In this case, we claim $C_{0}$ bounds a component $\Omega_{0}$ of $\Omega$. To see this, recall that $\Omega$ is a union of round disks with disjoint closures. The arc $J=g_{0}(-s, s)$ is the limit, along a subsequence, of $\operatorname{arcs} g_{n}\left(I_{n}\right) \subset \Omega$; since $\Omega$ has only finitely many components with diameter greater than $\operatorname{diam}(J) / 2$, there is a unique component $\Omega_{0}$ of $\Omega$ such that $g_{0}(0) \in \partial \Omega_{0}$. In fact the entire circular arc $J$ must lie in $\overline{\Omega_{0}}$, and hence $C_{0} \subset \overline{\Omega_{0}}$. Since $\left|C_{0} \cap \Lambda\right| \geq 2$, we have $C_{0}=\partial \Omega_{0}$.

Consequently the plane $H_{0}=\operatorname{hull}\left(C_{0}\right) \subset \mathbb{H}^{3}$ covers a component $S$ of $\partial \operatorname{hull}(M)$. In particular, we have $y \in \mathrm{T} S$.

Now even in this case, we have $s_{n} \rightarrow 0$ along the subsequence where $g_{n}(0) \notin \Omega_{0}$. Thus to complete the proof, it suffices to show that (2) holds under the assumption that $g_{n}(0) \in \Omega_{0}$ for all $n$. Under this assumption, $C_{n} \cap \Omega_{0}$ is a circular arc with two distinct endpoints, one of which is $g_{n}\left(s_{n}\right)$. Equivalently, $H_{n}=\operatorname{hull}\left(C_{n}\right)$ meets $H_{0}$ along a geodesic $\gamma_{n} \subset \mathbb{H}^{3}$, with one end converging to $g_{n}\left(s_{n}\right)$.

Let $\chi_{n} \subset H_{n}$ be the unique horocycle resting on $g_{n}(\infty)$. The natural lift of $\chi_{n}$ to $\mathrm{FH}^{3}$ gives the orbit $g_{n} U$. Let $\delta_{n}$ denote the geodesics in $\mathbb{H}^{3}$ connecting $g_{n}(\infty)$ to $g_{n}\left(s_{n}\right)$. Note that $\delta_{n}$ and $\chi_{n}$ both lie in the plane $H_{n}$, and cross at a unique point $p_{n}$.

We claim that $d\left(p_{n}, H_{0}\right) \rightarrow 0$. To see this, fix $\epsilon>0$. It is easy to see that the set of points in $H_{n}$ that are within hyperbolic distance $\epsilon$ of $H_{0}$ is convex and invariant under translation along $\gamma_{n}$; thus

$$
H_{n}(\epsilon)=\left\{p \in H_{n}: d\left(p, H_{0}\right)<\epsilon\right\}=\left\{p \in H_{n}: d\left(\gamma_{n}, p\right)<R_{n}\right\}
$$

for some $R_{n}>0$. Since $x_{n} \rightarrow \mathrm{id}$, we have $H_{n} \rightarrow H_{0}$ and hence $R_{n} \rightarrow \infty$; moreover, $\chi_{n}$ converges to a horocycle in $H_{0}$, so eventually $d\left(\gamma, \chi_{n}\right)<R_{n}-1$. By Lemma 4.2, this implies that $d\left(p_{n}, \gamma\right)<R_{n}$, and hence $d\left(p_{n}, H_{0}\right)<\epsilon$ for all $n \gg 0$. 
By construction we have $g_{n} u_{n} \in \mathrm{F}_{p_{n}} \mathbb{H}^{3}$. Since the frame $g_{n} u_{n}$ is tangent to the geodesic $\delta_{n}$, whose endpoints lie in the limit set, we have $\left[g_{n} u_{n}\right] \in$ RF $M$; and since $d\left(p_{n}, H_{0}\right) \rightarrow 0$ (and indeed $H_{n}$ and $H_{0}$ are nearly parallel near $\left.p_{n}\right)$, the frames $g_{n} u_{n}$ accumulate on $\mathrm{T} H_{0}$ and hence the frames $x_{n} u_{n}=$ $\left[g_{n} u_{n}\right]$ accumulate on $\mathrm{T} S$.

\section{Exceptional frames}

Let $M$ be a rigid acylindrical manifold. We define the locus of exceptional frames in $\mathrm{F} M$ by

$$
\mathrm{E} M=\bigcup\{x H V: x \in \mathrm{RF} M \text { and } x H \subset \mathrm{F} M \text { is closed }\} .
$$

In this section we develop some basic properties of the exceptional locus.

Immersed surfaces. As we remarked in $\S 1$, when $x \in \mathrm{RF} M$ and $x H$ is closed, its projection to $M$ is a properly immersed, totally geodesic surface $S$ passing through the convex core of $M$. For $v \in V$, the projection of $x H v$ to $M$ is a surface equidistant from $S$. The exceptional locus accounts for the all the horocycles that lie on such surfaces.

Like $\mathrm{RF}_{+} M$, the locus $\mathrm{E} M$ is invariant under the action of $A N$. In terms of the universal cover, we have $[g] \in \mathrm{E} M$ iff $g(\widehat{\mathbb{R}})$ is tangent, at $g(\infty)$, to a circle $C$ such that $|C \cap \Lambda| \geq 2$ and $\Gamma C$ is discrete. Note that

$$
\mathrm{E} M \cap \operatorname{RF} M \neq \emptyset
$$

since EM contains the compact $H$-orbits coming from the totally geodesic boundary components of the convex core of $M$.

Lemma 5.1 If $x \in \mathrm{RF} M$, then $\overline{x A U}$ meets $\mathrm{E} M$.

Proof. If $x H$ is closed, then we have $x \in \mathrm{E} M$ already. Otherwise, we have $\overline{x H}=\left(\mathrm{RF}_{+} M\right) H$ by Theorem 2.3, and $\overline{x A U} H=\overline{x H}$, since $A U \backslash H$ is compact. Thus $\overline{x A U} H=\mathrm{RF}_{+} M$ contains one of the compact orbits $y H \subset \mathrm{E} M$ coming from the boundary of the convex core of $M$, so $\overline{x A U}$ must meet this orbit as well.

Lemma 5.2 For any $x \in \mathrm{E} M \cap \mathrm{RF}_{+} M$, the locus $Y=\overline{x U}$ is a $U$-minimal set, and $Y=x v H v^{-1} \cap \mathrm{RF}_{+} M$ for some $v \in V$. 
Proof. Since $U$ commutes with the action of $V$, it suffices to treat the case where $x H$ is closed in FM. In this case, $x H=\mathrm{T} S$ for some properly immersed, totally geodesic surface $S \subset M$. The subgroup $\pi_{1}(S) \subset \pi_{1}(M)$ determines a covering space $M^{\prime} \rightarrow M$, which we can normalize so that $M^{\prime}=\Gamma^{\prime} \backslash \mathbb{H}^{3}$ with $\Gamma^{\prime} \subset H$. (If $S$ happens to be nonorientable, we pass to the orientation-preserving subgroup of index two.)

Since $S$ is properly immersed, $M^{\prime}$ is convex cocompact; and since $M$ is acylindrical, $M^{\prime}$ is nonelementary. It is now easy to check that the covering map $\mathrm{F}^{\prime} \rightarrow \mathrm{F} M$ sends $\left(\Gamma^{\prime} \backslash H\right) \cap \mathrm{RF}_{+} M^{\prime}$ isomorphically to $Y=(x H) \cap$ $\mathrm{RF}_{+} M$, respecting the action of $U$ (cf. [MMO, Thm. 6.2, Prop. 7.2]). The result then follows from Dal'bo's minimality Theorem 2.2.

Lemma 5.3 For any $x \in \mathrm{RF}_{+} M-\mathrm{E} M$, the orbit $x U$ meets $\mathrm{RF} M$.

Proof. Suppose $x \in \mathrm{RF}_{+} M$ but $x U$ does not meet RFM. Then $x=[g]$ where $C=g(\widehat{\mathbb{R}})$ meets $\Lambda$ in just one point. Therefore $C$ is tangent to $D=\partial \Omega_{0}$ for some component $\Omega_{0} \subset \Omega$, and $\Gamma D$ is discrete, so $x \in \mathrm{E} M$.

\section{Classification of $U$-orbit closures}

We can now complete the proof of Theorem 1.3. The interaction between $\overline{x U}$ and the exceptional locus EM plays a leading role in the proof.

Lemma 6.1 For any $x \in \mathrm{RF}_{+} M$, the orbit closure $X=\overline{x U}$ meets $\mathrm{E} M$.

Proof. Note that the result holds for $x$ if and only if it holds for some $x^{\prime} \in x A N$. Thus we are free to adjust $x$ by elements of $A N$ in the course of the proof.

Suppose $X$ is disjoint from EM. By Lemma 5.3, after replacing $x$ with an element of $x U$, we may assume $x \in \mathrm{RFM}$. Then $X$ contains a closed, $U$-invariant set $Y$ such that $Y L_{+} \subset Y$ for some 1-parameter semigroup $L_{+} \subset A V$, by [MMO, Prop. 9.3 and Thm. 9.4]. Let $L \subset A V$ be the group generated by $L_{+}$. Note that either $L=V$ or $L=v A v^{-1}$ for some $v \in V$.

Choose $\ell_{n} \rightarrow \infty$ in $L_{+} \cong \mathbb{R}_{+}$. Then $L=\bigcup \ell_{n}^{-1} L_{+}$. The locus $Y \ell_{n} \subset X$ is $U$-invariant, so by Lemma 5.3 again we can find $y_{n} \in \operatorname{RF} M \cap Y \ell_{n}$. Pass to a subsequence such that $y_{n} \rightarrow z \in \operatorname{RF} M$. We have $y_{n} \ell_{n}^{-1} L_{+} \subset X$ for all $n$, so in the limit we obtain $z L \subset X$. 
If $L=V$, then we have $z N \subset X$, so $X=\mathrm{RF}_{+} M$ by Theorem 2.1, and thus $X$ meets EM by equation (5.1). Otherwise, $L=v A v^{-1}$ for some $v \in V$. Therefore

$$
X \supset \overline{z v A U} v^{-1} \text {. }
$$

Again, we can find $u \in U$ such that $y=z u v \in \operatorname{RFM}$. Then $\overline{y A U}=\overline{z v A U}$. By Lemma 5.1, $\overline{y A U}$ meets E $M$, so $X$ meets E $M$ as well.

Typical orbits. Using the results of $\S 3$ and $\S 4$, we can now finally describe the behavior of $U$-orbits outside of the exceptional locus.

Theorem 6.2 Suppose $x \in \mathrm{RF}_{+} M-\mathrm{E} M$. Then $\overline{x U}=\mathrm{RF}_{+} M$.

Proof. Let $X=\overline{x U}$. Choose $y \in X \cap \mathrm{E} M$, using Lemma 6.1. By Lemma 5.2 , there is a $v \in V$ such that $Z=y v H v^{-1}$ is closed, we have

$$
X \supset Y=\overline{y U}=Z \cap \mathrm{RF}_{+} M,
$$

and $Y$ is a $U$-minimal set. Replacing $x$ with $x v$, we can assume that $v=\mathrm{id}$, and hence $Z=y H$. Then $Y \cap \operatorname{RF} M \neq \emptyset$, so we can also assume that $y \in \operatorname{RF} M$. By Lemma 5.3, after replacing $x$ with $x u$ for some $u \in U$, we can further assume that $x \in \mathrm{RF} M$.

Let $X^{*}=X \cap \operatorname{RF} M$, and let

$$
G_{0}=\left\{g \in G: Z g \cap X^{*} \neq \emptyset\right\} .
$$

We claim there is a sequence $g_{n} \rightarrow$ id in $G_{0}-H V$. To see this, first note that since $y \in X$, we can find $u_{n} \in U$ and $g_{n} \rightarrow$ id in $G$ such that $x u_{n}=y g_{n}$. In particular, we have $x u_{n} \rightarrow y$. We now apply Theorem 4.1. This Theorem implies that after changing our choice of $u_{n} \in U$, we can assume that $x u_{n} \in X^{*}$ and either (i) $x u_{n} \rightarrow y$, or (ii) $Z=Y$ is compact, and $x u_{n}$ accumulates on $Y$. In either case, after passing to a subsequence and (in case (ii)) possibly changing our choice of $y \in Y$, we still have $x u_{n}=y g_{n}$. Then clearly $g_{n} \in G_{0}$, we have $g_{n} \rightarrow$ id, and $g_{n} \notin H N=H V$ because $y H \subset \mathrm{E} M$ while $x \notin \mathrm{E} M$.

Since $Z$ is $H$-invariant, we have $H G_{0}=G_{0}$. By [MMO, Lemma 9.2], there is also a $K>1$ and a sequence of $K$-thick sets $T_{n}$ such that $g_{n} T_{n} \subset G_{0}$ for all $n$. Applying Theorem 3.1 (with the order of factors reversed) to the sequence $H g_{n} T_{n} \subset G_{0}$, we find that $G_{0}$ contains a thick subset $V_{0} \subset V$. In particular, we can choose $v_{n} \rightarrow \infty$ in $V \cap G_{0}$. Then $Z v_{n}$ meets $X^{*}$ by the definition of $G_{0}$. But $Z v_{n} \cap \mathrm{RF}_{+} M=Y v_{n}$, so the $U$-minimal set $Y v_{n}$ 
also meets $X^{*}$, and thus $Y v_{n} \subset X$ for all $n$. Now $Y v_{n}$ is invariant under the closed subgroup $v_{n}^{-1} A U v_{n}$ of $A N$, which converges to $N$ as $n \rightarrow \infty$. By compactness of $X^{*}$, we conclude that $X$ contains the $N$-orbit of a point in $X^{*}$, and hence $X=\mathrm{RF}_{+} M$ by Theorem 2.1.

Proof of Theorem 1.3. Let $x$ be an element of FM.

(1) If $x \notin \mathrm{RF}_{+} M$, then $x U$ is closed. Indeed, in this case $x U$ corresponds to a horocycle $\chi \subset \mathbb{H}^{3}$ resting on a point of $\Omega$, and the projection of $\chi$ to $M$ is a proper immersion.

(2) If $x \in \mathrm{E} M \cap \mathrm{RF}_{+} M$, then we $\overline{x U}=x v H v^{-1} \cap \mathrm{RF}_{+} M$ for some $v \in V$, by Lemma 5.2 .

(3) Finally, if $x \in \mathrm{RF}_{+} M-\mathrm{E} M$, then $\overline{x U}=\mathrm{RF}_{+} M$ by Theorem 6.2 .

\section{Classification of $A U-$ orbit closures}

In this final section we use the classification of $U$-orbits to show that

$$
\overline{x A U}=\overline{x H} \cap \mathrm{RF}_{+} M
$$

for all $x \in \mathrm{RF}_{+} M$, as stated in Corollary 1.5.

Generic circles. Let $M=\Gamma \backslash \mathbb{H}^{3}$ be a rigid acylindrical manifold. Let $\mathcal{C}=G / H$ be the space of circles in $\widehat{\mathbb{C}}$, let

$$
\mathcal{C}_{0}=\{C \in \mathcal{C}:|C \cap \Lambda| \geq 2\}
$$

and let

$$
\mathcal{C}_{1}=\left\{C \in \mathcal{C}_{0}: \Gamma C \text { is discrete in } \mathcal{C}\right\} .
$$

Lemma 7.1 The set $\mathcal{C}_{1}$ is countable.

Proof. A circle $C \in \mathcal{C}_{1}$ corresponds to a properly immersed, totally geodesic surface $S$ with fundamental group $\pi_{1}(S) \cong \Gamma^{C}$. Thus $\Gamma^{C}$ is a finitely generated, nonelementary group and $C$ is the unique circle containing $\Lambda\left(\Gamma^{C}\right)$.

Since $\Gamma$ is countable, there are only countably many possibilities for $\Gamma^{C}$, and hence only countably many possibilities for $C$. 
Lemma 7.2 There is a circle $C \in \mathcal{C}_{0}$ that is not tangent to any circle in $\mathcal{C}_{1}$

Proof. It is easy to see that $\mathcal{C}_{0}$ has nonempty interior, while the set of circles tangent to a given $C \in \mathcal{C}_{1}$ is nowhere dense. Since $\mathcal{C}_{1}$ is countable, the result follows from the Baire category theorem.

Rephrased in terms of $\Gamma \backslash G$, this shows:

Corollary 7.3 There is an orbit $y H \subset \mathrm{F} M-\mathrm{E} M$ that meets $\mathrm{RF} M$.

Proof of Corollary 1.5. The argument is similar to the proof of Lemma 5.1. Consider $x \in \mathrm{RF}_{+} M$. We always have $\overline{x A U} \subset \mathrm{RF}_{+} M$, since the latter set is closed and $A U$ invariant.

If $x U$ meets $\mathrm{RF} M$, then we can reduce to the case where $x \in \mathrm{RF} M$. Under this assumption, if $x H$ is closed, then $\overline{x U}=\overline{x H} \cap \mathrm{RF}_{+} M$ by Theorem 1.3; since the latter set is $A$-invariant, it also coincides with $\overline{x A U}$. Otherwise, by Theorem 2.3 and compactness of $A U \backslash H$, we have

$$
\overline{x A U} H=\overline{x H}=\mathrm{RF}_{+} M .
$$

In particular, by Corollary $7.3, \overline{x A U}$ meets $\mathrm{RF}_{+} M-\mathrm{E} M$. Let $y$ denote a point in their intersection. Then we have

$$
\mathrm{RF}_{+} M=\overline{y U} \subset \overline{x A U}
$$

by Theorem 6.2 , so equation (7.1) holds in this case as well.

Finally, if $x \in \mathrm{RF}_{+} M$ but $x U$ does not meet $\mathrm{RF} M$, then $x H$ corresponds to a circle tangent to $\Lambda$ in just one point, and (7.1) is easily verified using minimality of the horocycle flow on a compact hyperbolic surface (cf. [MMO, Theorem 1.5]).

\section{References}

[Da] F. Dal'bo. Topologie du feuilletage fortement stable. Ann. Inst. Fourier 50(2000), 981-993.

[Fer] D. Ferte. Flot horosphérique des repères sur les variétés hyperboliques de dimension 3 et spectre des groupes kleiniens. Bull. Braz. Math. Soc. 33(2002), 99-123. 
[MMO] C. McMullen, A. Mohammadi, and H. Oh. Geodesic planes in hyperbolic 3-manifolds. Preprint, 2015.

[Rn] M. Ratner. Raghunathan's topological conjecture and distributions of unipotent flows. Duke Math. J. 63(1991), 235-280.

[Sh] N. A. Shah. Unipotent flows on homogeneous spaces of $\operatorname{SL}(2, \mathbb{C})$. M.Sc. Thesis, Tata Institute of Fundamental Research, Mumbai, 1992.

[Win] D. Winter. Mixing of frame flow for rank one locally symmetric spaces and measure classification. Israel J. Math. 210(2015), 467507. 